For reprint orders, please contact: reprints@futuremedicine.com

\title{
Interview with Professor Kay Davies
}

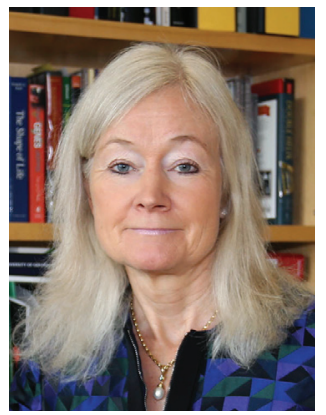

\section{Kay Davies* speaks to Ellen Clarke, Commissioning} Editor: Kay Davies is the Dr Lee's Professor of Anatomy in the Department of Physiology, Anatomy and Genetics, and Director of the MRC Functional Genomics Unit at the University of Oxford, UK. Her research interests lie in the molecular analysis and development of treatment for human genetic disease, particularly, Duchenne muscular dystrophy (DMD) and the application of genomics for the analysis of neurological disorders and gene-environment interactions. She is co-founder of a company, Summit Therapeutics, which aims to deliver utrophin modulation to the clinic for the therapy of DMD, which currently has a drug in Phase 1 trials. She has published more than 400 papers and won numerous awards for her work. She is a founding fellow of the UK Academy of Medical Sciences and was elected a Fellow of the Royal Society in 2003. She has been a Governor of the Wellcome Trust since 2008 and became Deputy Chairman in October, 2013. She was made Dame Commander of the British Empire for services to science in 2008.

\section{Q How did you first become involved in the field of muscular dystrophy research?}

I first became involved in muscular dystrophy research while working on another more common disorder called cystic fibrosis in Bob Williamson's Laboratory in London. There was no prenatal test for $\mathrm{CF}$ and nothing was known about the underlying cause, much the same as the situation with DMD. However, for DMD we knew that the gene at fault must lie on the $\mathrm{X}$ chromosome since this disease usually only affects boys. Boys have an X and $\mathrm{Y}$ chromosome but girls have two $\mathrm{X}$ chromosomes; the normal $\mathrm{X}$ chromosome can compensate for the defective gene on the other $\mathrm{X}$ in girls. We could, therefore, develop new methods to search for a gene on one chromosome rather than search for the CF gene amongst the 22 nonsex chromosomes. It was then that the Executive Director of the Muscular Dystrophy UK charity approached us to work on DMD. We worked closely with clinical colleagues in Cardiff to collect families, to identify the exact position of the faulty gene which led to the first prenatal test for this devastating disease. It was during this period that I met patient groups, patients and their families and I recognized the unmet need, not just for a test for this disease, but also for a therapy.

\section{Q What have been your most} significant contributions to this area of neuroscience?

When I became involved in DMD, nothing was known about the cause, no prenatal test was available and carrier testing was unreliable. Although the disease ran in families, new mutations were common where affected boys were diagnosed without any family history. In families where the disease was identified, some mothers opted to abort all boys, such was the severity of the condition. Our first significant contribution came from the development of a prenatal test and with it a reliable carrier test. Kunkel in Boston identified the mutated gene first and we worked with his group and others round the world to show that most mutations are deletions

*Department of Physiology, Anatomy \& Genetics, MRC Functional Genetics Unit, University of Oxford, UK; kay.davies@dpag.ox.ac.uk 
of the gene. The gene turned out to be the largest in the human genome encoding a large structural protein, dystrophin. This is too large to fit into most viruses used in gene therapy protocols. We identified one patient in particular who was mildly affected and yet had half his gene missing. We demonstrated that delivery of a minigene based on this patient's mutation could rescue the pathology in the mdx mouse model of the disease. This minigene and other versions of it are now used by many groups around the world in gene therapy protocols that are now moving into the clinic.

We also discovered a protein, utrophin, which is very similar to dystrophin. In adult muscle, utrophin is present at the nerve muscle junction with dystrophin at the muscle membrane, but in early human development both proteins are together at the muscle membrane. We have shown that increasing the levels of utrophin in adult muscle can prevent the pathology in the mdx mouse and have identified a drug that can increase utrophin expression. This drug, SMT C1100, was developed with Summit Therapeutics and is currently in a Phase $1 b$ trial in DMD patients.

\section{What are the main areas of research in} your laboratory at present?

The main areas of research in my laboratory at the moment are the development of a therapy for DMD and the analysis of gene involved in behavior and neurodegeneration. I have already mentioned our commitment to utrophin modulation with Summit Therapeutics.

The other strand of the research program builds on our initial observational testing of mouse mutants from which we identified lines that could potentially provide insights into neurodegenerative diseases and schizophrenia. We have shown that increases in the expression of a gene involved in oxidative stress can ameliorate ALS and demonstrated that abnormal circadian rhythms are associated with a schizophrenia endophenotype through a defect at the synapse. We have also characterized a novel lipodystrophic neurological mutant as the first model for the fat malabsorption disorder known as chylomicron retention disease, which results in defects in lipid transport and storage and demyelination in the nervous system.

\section{Q How important are transgenic animal models to your research?}

Transgenic animals have been vital for the progress in the understanding of the pathology of the disease. It is very difficult to access muscle samples from patients as muscle biopsies are painful and only a small amount of muscle can be obtained at each sampling. Mouse models provide a supply of all types of muscle so that any potential therapy can be assessed before administration in man. Transgenic animals are necessary for understanding of the molecular mechanisms that lead to the disease as well as testing the efficacy of any drug. It is important to understand the mechanism of action of any therapy, to determine possible dosage regimes, and above all to test safety before drugs are delivered to the patients. These data in transgenic animals are needed before the regulatory authorities will give the go-ahead for clinical trials.

\section{Q Can you tell us a little about your interest} in the ethical implications of your research? I have always been interested in the ethical implications of my research. In the early days of genetic testing, I was very much involved in the implications for insurance and the need for confidentiality for patients and their families. I served on the Advisory Group on Genetic Testing set up by the UK government which discussed these issues as well as the presymptomatic testing for late onset diseases such as Parkinson's disease and Alzheimers. There were individuals on the Committee that believed families should be able to choose which disorders the unborn child should be tested for, and they also believed that this should include the right to choose the sex. Others believed that tests should only be carried for severe genetic disorders. It was an interesting debate that included patient groups as well as other lay individuals. Fortunately, today there is less fear about insurance companies discriminating against those with a family history of genetic disease as there is more understanding of the risks. It remains very important to ensure that the public are aware of the risks and benefits of genetic research.

\section{Q Currently what are the most promising genetic approaches to muscular dystrophy therapy?}

There are numerous pharmacological treatments being applied to muscular dystrophy that slow the disease process down but many of these will have long-term side effects and a life-long treatment will be needed. However, there are genetic approaches that are now moving to the clinic. The delivery of minidystrophin genes to the arm of patients using 
a virus has been undertaken. A limited immune response to dystrophin was observed but this could potentially be overcome by transient immunosuppression. The challenge with this approach is the targeting of all muscle, including the heart. Other approaches skip the mutation. Translarna (PTC Therapeutics) is now in the clinic which promotes the readthrough of stop codons that underly the disease in about $12 \%$ of patients. The efficacy of this drug remains to be determined. Another approach skips over the mutations and results in a smaller, partially functional dystrophin. This is currently being taken to the clinic by Sarepta Therapeutics and BioMarin Inc. for exon skipping of exon 51, a strategy that can be used in $13 \%$ of patients. In our strategy to modulate the expression of utrophin, Summit Therapeutics is testing a drug, SMT C1100 in a Phase $1 \mathrm{~b}$ trial. This approach has the advantage of being applicable to all patients whatever their mutations and being able to be delivered to the heart as well as skeletal muscle.

Q Can you tell us about some of the clinical trials that are currently in process? Are there any novel treatments on the horizon?

Most DMD boys have been treated with steroids or drugs that increase muscle mass to slow the progression of the disease. These drugs have side effects and, therefore, are not standard care in all clinics worldwide. However, a new generation of steroids is being tested that potentially do not have the side effects. These could be used alongside the genetic therapies in the future.

Muscle stem cells are also being developed. One clinical trial has been carried out but showed very low efficiency in delivery to muscle. This is an exciting approach, but much more work needs to be done in understanding the behavior of stem cells, in growing them up and then finally in targeting them to the right place and to all muscle.

\section{Q What are the greatest challenges in the field?}

Forty percent of our body weight is muscle and DMD patients show not only defects in skeletal muscle but also significant problems in the heart. An effective therapy needs to target all muscle and the treatment would need to be life-long. It is important, therefore, that any new drug has a very good safety profile.

DMD is a devastating disease and there is considerable unmet need. In recent years the regulatory authorities have recognized this and worked with patient groups and companies to accelerate development of new treatments while protecting the safety of the patient.

\section{Q What are your future research plans?}

My future research plans are focused on the optimization of the utrophin modulation approach for the therapy of DMD. It is rare that the first drug in a class is the best in class so we are optimizing conditions for the treatment with SMT C1100 because it has an excellent safety profile in patients in addition to beneficial effects in preclinical models. Concurrently we are developing follow-on molecules that will increase utrophin levels and potentially be even more potent therapeutics. In the longer term we will test this approach in combination with exon skipping to provide maximum benefit for patients.

\section{Q How do you see the field progressing in} the next 5 years?

In DMD, the next 5 years will witness the best advances in treatment yet. I think there will be better constructs for viral therapy, targeting of more than exon 51 in the exon skipping strategy and better chemistries to improve efficiency of delivery, other molecules that may promote stop codon readthrough in addition to Translarna, and drugs that increase the levels of utrophin for clinical benefit. All of the treatments will work optimally if administered at birth, and therefore newborn screening will need to be introduced. Nevertheless, many of these therapies will still be able to slow the progression of the disease and improve quality of life in adult patients in a wheelchair. I am convinced that we are now into a breakthrough phase for the therapy of DMD.

\section{Disclaimer}

The opinions expressed in this interview are those of the interviewee and do not necessarily reflect the views of Future Medicine Ltd.

\section{Financial \& competing interests disclosure}

Kay E Davies is a shareholder of Summit Therapeutics and on the Scientific Advisory Board of Summit Therapeutics and Prosensa. The author has no other relevant affliations or financial involvement with any organization or entity with a financial interest in or financial conflict with the subject matter or materials discussed in the manuscript apart from those disclosed.

No writing assistance was utilized in the production of this manuscript. 